\title{
Aggressive Fibromatosis Treatment - Imatinib Challenges
}

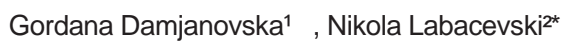 \\ 1 REPLEK FARM, Kozle 188, Skopje, Republic of Macedonia; Institute for Preclinical and Clinical Pharmacology and Toxicology, Medical \\ faculty, University "Ss Ciryl and Metodius", 50 Divizija 16, Skopje, Republic of Macedonia
}

Citation: Damjanovska G, Labacevski N. Aggressive Fibromatosis Treatment - Imatinib Challenges. Maced J Med Sci. 2012 Oct 15 5(3):346-355 http://dx doi.org/10.3889/ MJMS.1857-5773.2012.0262.

Key words: aggressive fibromatosis; desmoid tumors; imatinib.

Correspondence: Nikola Labacevski. Institute for preclinical and clinical pharmacology and toxicology, Medical faculty, University"St Ciryl and Metodius", 50 Divizija 16, Skopje, Republic of yahoo.com

Received: 02-Jun-2012; Revised: 10-Jun-2012; Accepted: 23-Sep-2012; Online first: 08-Oct-2012

Copyright: ๑ 2012 Damjanovska G. This is an open-access article distributed under the terms of the Creative Commons Attribution License, which permits unrestricted use, distribution, and author and source are credited.

Competing Interests: The author have declared that no competing interests exist.

\begin{abstract}
Background: Desmoid tumor lately referred as aggressive fibromatosis (AF) is rare clinically heterogeneous and unpredictable disease with prevalence $<0.03 \%$ of all malignancies. The current standard treatment is: radical surgical treatment with tumour excision accompanied by radiotherapy and/or systemic approaches. The potential morbidity and the high local recurrence rates leaded investigators to assess the role of pharmacological approaches. The purpose of this review was to overview effectiveness of treatment options for $\mathrm{AF}$ and the role of imatinib mesylate (Glivec ${ }^{\mathrm{TM}}$; Novartis), in $\mathrm{AF}$ that has been published over the past decade.
\end{abstract}

Material and Methods: Published clinical trials, studies, case reports and case series literature review, was carried out using the electronic databases PubMed/Medline from 2002 to 2012.

Results: Reviewed literature included case reports, case series, retrospective studies and several small phase II trials. According to the review NSAIDs treatment performed positive response range of $37 \%$ $57 \%$, COX 2 treatment (Meloxicam) up to $90 \%$ response, treatment with hormonal agents response ratio between $40 \%-50 \%$, positive responses for chemotherapy against AF of almost $50 \%$ and response rate for Imatinib 10\%. However several questions need to be answered: which is the most suitable treatment at certain AF condition, what is the optimal dose and duration of treatment since the lack of sufficient patient numbers and randomized trials compromises the validity of the reported results.

Conclusions: Reviewed literature presents evidence that citotoxic and non-citotoxic systemic therapies other that surgery are effective against AF. Main issue that has to be processed is stronger validation of results by further investigation with precisely designed prospective studies with larger patient numbers and, with main end points that include tumor respones rate and survival as well as quality of life.

\section{Introduction}

According to the World Health Organization, desmoid tumors are defined as "clonal fibroblastic proliferations that arise in the deep soft tissues and are characterized by infiltrative growth and a tendency toward local recurrence but an inability to metastasize." Aggressive fibromatosis (AF), also used as synonym term for desmoid tumor in its variable biological behavior is classified between benign fibrous tissue proliferation and fibrosarcoma [1]. Since these tumors have a strong potential for local invasiveness, high recurrence rates, tendency toward destruction of the surrounding tissue and significant morbidity and potential mortality, they are classified as semi-malignant. AF is rare disease: accounts for $<3 \%$ of all soft tissue sarcomas and approximately $0.03 \%$ of all malignant disease. General population estimated incidence is $2-4$ per million per year. AF 
occurs between the age of 15 and 60 years with a peak age of about 30 years, more in women than in men and may affect all sites, including the extremities, trunk, and abdomen [2]. Aetiology of these tumors is multifactorial: genetic, endocrine, and physical factors have been stated. AF arises either sporadically (extra-abdominal) or in association with the familial adenomatous polyposis (FAP) and Gardner syndrome (intra-abdominal, mainly mesenteric) [3]. On the molecular level, patients with FAP express germ-line inactivating mutations of the adenomatous polyposis coli (APC) gene [4], whereas patients with sporadic $A F$ usually express somatic betacatenin (CTNNB1) activating mutations [5]. APC and beta-catenin are members of the WNT pathway which leads to conclusion that WNT pathway is possibly altered in all aggressive fibromatoses irrespective of familial or sporadic origin. AF clinically is highly heterogeneous and unpredictable: including phases of progression and growth, stabilization, and sometimes spontaneous regression. At the moment, possible correlation between either beta-catenin or APC mutations and disease penetrance or recurrence exists. Stabilization of disease progression and spontaneous regression are even more difficult to explain, and those may be due to modifying factors of the microenvironment and host's humoral millieu.

Due to variable clinical presentation and behaviour, no standard approach for AF can be recommended. The treatment of choice for these tumors has been changing and may involve current standard: radical surgical treatment with tumour excision accompanied by radiotherapy and/or systemic approaches. The potential morbidity of surgery and radiation therapy and the high local recurrence rates have lead investigators to assess the role of pharmacological approaches in settings in which surgery and radiation therapy are either not possible or unsuccessful.

In this review article, we collect and analyze all available information regarding effectiveness of treatment options for AF, with focus on targeted therapy with tyrosine kinase inhibitor imatinib mesylate.

\section{Material and Methods}

For the purposes of this review we obtained, review and analyzed all available data for AF in the English language literature. Through a search of the electronic databases PubMed/ Medline we identified published trials, studies and series. The keywords 'aggressive fibromatosis', 'desmoid tumors' and 'imatinib' were used. Cross-referencing, using the references of identified studies, were used in addition to the computerised searches. Inclusion criteria for these review were cases with information about patients' age and sex, tumor status (primary versus recurrent), previous therapy, type of therapy, response and duration of response.

\section{Results}

\section{Surgery}

Surgery has been traditionally considered the mainstay treatment. This particularly applies for patients with primarily resectable and localized tumors where standard goal is complete resection with negative microscopic margins. In one of the largest series of 234 patients with extra-abdominal AF treated for over 35 years, only $17 \%$ of the patients had a recurrence and $23 \%$ of the patients needed additional surgery [6].

However, the growth pattern of these tumors is deep infiltrating, and there is no tumor capsule. Because the boundaries of the tumors are difficult to distinguish intra operatively from scars or connective tissue, R0 resection is not always possible. Also, R0 resection is not always possible because of anatomic boundaries. In addition, because of variability in the clinical course and the importance of site involvement, the application and use of surgical intervention have been extensively discussed $[7,8]$. Mostly considered variables associated with local recurrence are those including surgical margin status which is complex. For tumor-free and tumorpositive margins, response rates of $72 \%$ and $41 \%$, respectively, and local control rates of $94 \%$ and $75 \%$, respectively, have been reported [9].

Despite the results, the importance of a negative surgical margin is debated. Massachusetts General Hospital (MGH) study [11] and study from the M.D. Anderson Cancer Center (MDACC) [10] revealed that microscopically positive margins significantly influenced local recurrence rates. At the same time, Memorial Sloan-Kettering Cancer Center (MSKCC) [16], Instituto Nazionale Tumori [13], and recent MDACC [14] studies have failed to demonstrate any such significance of surgical margin on recurrence. In addition, a recent study composed of the largest series of sporadic desmoid tumors also showed that microscopic assessment of surgical resection quality (R0 vs. R1) did not have a significant impact on progression-free survival [24]. Also, in a long-term follow-up of 89 patients, it turned out that 
patients with microscopically complete surgery had an event-free survival rate similar to that of patients undergoing non surgical strategies [18]. Finally, despite the use of surgery $20-36 \%$ of patients will show local recurrence [19] that, although classified as benign, can result in death due to local destruction in a small but important fraction of patients. It must be noted that spontaneous regressions have also been reported, a fact that supports a wait-and-see policy after resection without wide margins [7]

Taking into consideration all these heterogeneous results, surgical therapy must be performed to what is achievable in terms of margins while preserving functional status for the individual patient. Attempts to achieve negative margins may result in unnecessary morbidity and may not definitively prevent local recurrence. The consequences of radical excision may be worse than the disease itself [20].

\section{Radiotherapy}

Radiotherapy in the treatment of AF has been used either as adjuvant therapy to surgery with positive margins or as primary therapy for extra-abdominal tumors that are unresectable or where surgery derived functional/ cosmetic loss is unacceptable.

Comparative review of 22 articles in period from years 1983-1998[21] analyzed results from surgery treatment alone, surgery with radiotherapy, or radiotherapy treatment alone (dose range, 10-75 Gy). Mean follow-up for recurrences in 780 patients ranged between 2.0 and 10.4 years. The results show significantly better local control when radiotherapy alone (78\%) and surgery combined with radiotherapy (75\%) was applied than when surgery alone (61\%) was performed. Relapse rate when radiation therapy was added postoperatively in patients with positive surgery margins, decreased from $59 \%$ to $25 \%$. These results were reported for primary as well as for recurrent AF. The radiation therapy complication rate was $22.8 \%$, and tissue fibrosis was the most common complication. The most serious risk that has to be noted is radiation induced malignancy [22].

In contrast to these results retrospective analysis of clinical data for 95 patients with AF who underwent therapy at the University of Michigan from 1984 through 2008 suggest equivalent local control rates between the groups receiving only surgery, only radiation, and a combination of both [23].

Newly suggested approach to increase the resectability and reduce rates of local recurrence in extra-abdominal desmoids is the use of preoperative radiotherapy [6]. However, confirmation with larger prospective randomized trials is needed.

Soft Tissue and Bone Sarcoma Group of the European Organization for Research and Treatment of Cancer (EORTC) performed pilot study (EORTC 62991) assessing moderate-dose radiotherapy for aggressive fibromatosis in patients not amenable to resection without significant function loss to evaluate the efficacy of radiotherapy. Forthy patients (last recruitment date April 2008) in this nonrandomized, phase II study received radiotherapy for a total of $56 \mathrm{~Gy}$ in 28 fractions. Patients are under follow up until final analysis is published.

\section{Noncytotoxic agents}

Antiinflamatory agents: NSAIDs use in the treatment of AF was based on the observation of the total regression of a single recurrent desmoid tumor of the sternum in a patient taking indomethacin for radiation induced pericarditis [24]. A variety of NSAIDs have been tested either alone or in combination with hormonal agents such as tamoxifen and testolactone [25]. NSAIDs, such as sulindac or indomethacin, demonstrated 37\%$57 \%$ responses, either as partial or complete response in several non-randomized retrospective studies [19]. Tsukada et al. assessed the efficacy of sulindac in 14 patients with a history of recurrent abdominal desmoid tumors [26]. The overall response rate was 57\% with delayed response mean time of 24 months. The rationale for NSAIDs use for AF treatment is based on evidence that COX-2 plays role in the growth of desmoid tumors with pharmacologic blockade of COX resulting in decreased cell proliferation in desmoid cell cultures in vitro, and COX-2 blockade resulting in smaller desmoid tumors in an in vivo mouse model. Poon et al. [27] showed that COX-2 is expressed in the majority of desmoid tumors, and Signoroni et al. showed that COX2 protein and mRNA were over expressed in all of their 14 cases [28]. There have been few prospective reports analyzing the effect of a COX-2 inhibitor, against desmoid tumors. Francis et al. reported an analysis of 52 patients with resectable desmoid tumors, in which 16 patients were treated with tamoxifen and cereblex treatment for 1.0 year, with $50 \%$ response, either as partial or complete response [29]. Recently Nishida et al. reported results from prospective study comprising 20 patients with extra-abdominal fibromatosis successfully treated with meloxicam 10mg daily. According to Response Evaluation Criteria in Solid Tumors (RECIST) [30] nineteen of the 20 patients (95\%) were evaluated as final 
status with equal or better than stable disease. The median period of medication was 36 months (range, 381 months) at 2011 [31].

Hormonal agents: Antihormonal therapy use for the treatment of desmoid tumors is based on observations of the natural history of the disease: about $80 \%$ of agressive fibromatosis occurs in women, incidences of fibromatosis is higher during and after pregnancy and spontaneous tumor regression after menopause have been reported. Studies have shown that presence of antiestrogen binding sites (AEBS) distinct from estrogen receptors (ERs) probably plays an important role in modulating or mediating the action of antiestrogens. ER-negative desmoid tumors with presence of high levels of AEBS might explain responses to tamoxifen when the overall incidence and concentration of ERs is low in these tumors. One of the most commonly used antiestrogens in AF is tamoxifen, many times referenced in the literature, mostly single case reports (dose range, 20-80 mg daily) presenting partial response or disease stabilization, that disables firm conclusion regarding the effectiveness of tamoxifen given as single agent against AF. It was reported, in a nonrandomized setting, that high-dose tamoxifen (120-200 mg daily) combined with sulindac $300 \mathrm{mg}$ daily may be more effective (positive response 52\%) than only lower doses of tamoxifen 10-40 mg daily [32]. There are no randomized data supporting the use of high doses of tamoxifen, and the risk for second cancers and deep venous thrombosis could be greater with its use.

A number of other hormonal agents have been tested and found to be effective in AF: toremifene, progesterone, medroxyprogesterone acetate, prednizolone, testolactone and gosereline [33-36].

Toremifene appear to be effective against desmoids as first- and second-line therapy. Brooks and co-workers [33] treated $20 \mathrm{AF}$ patients with toremifene at an average dose of $200 \mathrm{mg} /$ day. When used as first-line therapy the overall response rate was 50\%, while toremifene as second-line therapy gave a response rate of $33 \%$.

Testolactone acts by irreversibly inhibiting aromatase. It is proposed that testolactone acts through inhibition of cyclic AMP synthesis, which is known to influence growth and proliferation of fibroblasts. The largest reported study on the activity of testolactone is that of Waddell and Kirsch in a series of 17 patients [36]. Testolactone administered as a single agent $750 \mathrm{mg}$ daily gave an overall response rate of $40 \%$.

\section{Cytotoxic chemotherapy}

Chemotherapy with cytotoxic agents represents an appropriate choice for patients with unresectable, rapidly growing tumors or for patients who are highly symptomatic and/or with life-threatening desmoid tumor.

There have been no single-arm trials utilizing single-agent therapy in the literature. Several combination chemotherapy regimens are active with doxorubicin, liposomal doxorubicin, dacarbazine, ifosfamide, and methotrexate plus vinca alkaloid producing significant responses, according to the RECIST, ranging from $50 \%$ to $80 \%$ (partial responses or stable disease for at least 6 months) [37].

Constantinidou A at al. [38] have reported significant activity of pegylated liposomal doxorubicin applied to 12 patients at a dose of $50 \mathrm{mg} / \mathrm{m} 2$ every 4 weeks, with four(33\%) partial response and seven (58\%) stable disease. Although with lower toxicity compared to parent drug, dose reductions are appropriate especially when the approach is to give therapy to a maximum response, which can take 12-18 months or even longer.

Recent retrospective study conducted by de Camargo et al. collected data over 15 years recommended administering anthracyclines. As reported [39], from seven different lines of treatment, anthracyclines appeared to be the most active: when applied to 35 patients anthracycline-based therapy resulted in 13 (37\%) partial responses and 18 (51\%) stable disease.

Garbay et al. [40] recently updated the results of chemotherapy for AF in the French Sarcoma Group study that enrolled 62 patients and in most of them several other treatments has failed. Forty four patients (71\%) were treated with combination chemotherapy. Overall, 80\% responded: complete response 1 (1.6\%), partial response 12(19.4\%), stable disease 37 (59.6\%) The response rate was higher with anthracyclinecontaining regimens: The only factor identified predictive of a shorter interval to progression was non limb location.

Methotrexate with vinblastine administered on a weekly basis was proposed as a less toxic alternative to doxorubicin based and VAC chemotherapy. Skapek [41] reported that the combination was effective in study including 27 patients, with overall response rate 66\%: 8 (29.6\%) partial responses and 10 (37\%) stable disease. Azarrelli et al. conducted methotrexate with vinblastine study enrolling 27 patients with overall response rate 85 , 18\%, 4 (14.81\%) partial responses and 19 (70.37\%) 
stable disease. Study demonstrated that weekly treatment was not feasible due to myelotoxicity and hepatotoxicity and the mean interval between cycles was found to be 15 days [42]. Substitution of vinorelbine for vinblastine resulted in significantly less neurotoxicity without a compromise in response rates.

Conventional chemotherapy, typically administered in low doses over a prolonged period of time, has demonstrated encouraging results; however, the duration of treatment is inherently limited by cumulative toxicity.

\section{Imatinib}

Imatinib mesylate is a selective protein-tyrosine kinase inhibitor that inhibits the bcr-abl tyrosine kinase and is also an inhibitor of other class 3 receptor tyrosine kinases: receptor tyrosine kinases for platelet-derived growth factor (PDGF) and stem cell factor (SCF), c-kit (CD117), and inhibits PDGF and SCF mediated cellular events. Lately it was reported that imatinib is inhibits macrophage-colony stimulating factor receptor (MCSFR) too. This agent blocks ligand activated receptor phosphorylation and mitogen-activated kinase activation and proliferation, resulting in the inhibition of cellular growth and proliferation.

FDA granted approval for advanced gastrointestinal stromal tumors which express an activating c-kit in 2002. The same year, two patients with unresectable AF and progressive disease, were treated by Mace and colleagues and due to reported dramatic responses to imatinib [43], amputation as final surgical solution was avoided. Thus expression of PDGFR-A, PDGFR-B and CKIT leaded to assumption of possible response to imatinib in AF patients that encouraged its use by physicians.

Following experience of Mace and colleagues several other cases were reported including patients with AF previously treated with surgery, radiotherapy and different systemic approaches all resulting with recurrent AF. Therapeutic regimes included imatinib $400 \mathrm{mg}$ to $600 \mathrm{mg}$ daily with positive responses after 7-11 months of administration. Additionally, possible correlation between expression and mutation of $\mathrm{c}$-Kit and PDGFRs and positive response to imatinib in patients with AF was hypotetized.

In order to determine the clinical efficacy of imatinib in patients with advanced AF heavily pretreated with different therapeutic approaches and to identify the molecular basis of response/nonresponse to imatinib,
Heinrich et al. [44] treated 19 patients with $800 \mathrm{mg}$ imatinib daily. Three of 19 patients $(15.7 \%)$ had a partial response to treatment lasting longer then 1, 5 years, and four patients (21\%) having stable disease that lasted more than 1 year (overall 1 year tumor control rate of $36.8 \%)$. One of the patients with partial response had objective response that lasted more that 3 years. Authors did not find any mutations of c-Kit, PDGFR-A, or PDGFR$B$, but reported that sixteen of 19 patients (84\%) had mutations involving the WNT pathway (APC or betacatetin). However, there was no correlation between WNT pathway mutations and clinical response to imatinib. The authors reported that plasma level of PDGF-BB was inversely correlated with time to treatment failure.

Same author reported results from Phase II, open-label study evaluating the activity of imatinib in treating life-threatening malignancies known to be associated with imatinib-sensitive tyrosine kinases. One hundred eighty-six patients with 40 different malignancies that had proven refractory to standard therapy were enrolled in this study and treated with imatinib $800 \mathrm{mg}$ daily. Two of 20 patients with AF enrolled in this study (10\%) had a partial response and eight patients (40\%) had stable disease with median time to progression of 9 , 1 month. [45]

All 20 patients were c-kit negative and PDGFR$B$ positive that arouse conclusion that detection of c-kit by immunohistochemistry is not necessarily predictive of a response to imatinib therapy and that all responses to imatinib included neoplasm for which activation of an imatinib-sensitive tyrosine kinase (predominantly PDGFR) occured via genomic mutation and/or rearrangement. Authors reported that it is not clear if the activity of imatinib in these tumors is due to inhibition of imatinib-sensitive tyrosine kinase signalling in tumors or occurs by other mechanisms.

Phase II Multicenter Sarcoma Alliance for Research through Collaboration (SARC) Trial to investigate the outcome of AF patients treated with imatinib presented promising response rates [46]. As reported by Chugh $\mathrm{R}$ et al. fifty-one AF patients with or without previous treatment and locally advanced disease were enrolled in the study. Patients were dosed according to body surface area and received from 200-600mg imatinib. In this phase II trial, RECIST response rate for imatinib is <10\%: among 51 patients, 3 (6\%) had an objective response-partial response. The progression free survival rate at 2 and 4 months was $94 \%$ and $88 \%$, respectively, and $66 \%$ and $58 \%$ at 1 year and 3 respectively. 
Laboratory evaluation of tissue specimen presented immunohistochemical positivity for c-kit, PDGFR-a, PDGFR-b, AKT, Phosphatase and betacatenin, and mutational changes for c-kit, PDGFR-a, PDGFR-b and beta-catetin.

However, these findings did not show any significant correlation with outcome or response.

Penel et al. [47] conducted FNCLCC/French Sarcoma Group phase II trial with a long-term follow-up to evaluate efficacy of imatinib as a new treatment option in patients with recurrent or established progressive aggressive fibromatosis.

Forty patients with unresectable and progressive symptomatic AF previously undergone different treatments were treated with imatinib $400 \mathrm{mg}$ daily for 1 year. At 3 months evaluation of response rate in 35 patients amendable to radiological review presented: one $(2.9 \%)$ complete, three $(8.6 \%)$ partial responses and $28(80 \%)$ stable disease. The non-progression rates at 3,6 , and 9 months were, respectively, $91 \%, 80 \%$ and $66 \%$. The 1 and 2 -year progression-free rates were $67 \%$ and $55 \%$ respectively, while overall survival rate was 95\%. This trial did not include identification of expression and/or mutations in imatinib sensitive tyrosine kinase, so Penel at al. are currently performing biological studies on plasma and tumor specimens collected during the study to better identify the tyrosine-kinase dependent pathway implied in AF.

Other studies investigated the prognostic value or influence of different c-kit mutations in the response to imatinib [48-50].

Currently, German Interdisciplinary Sarcoma Group conducts clinical phase II study to evaluate the role of imatinib and nilotinib in the induction of progression arrest in patients with aggressive fibromatosis with documented progression and not amenable to surgical R0 resection or accompanied by unacceptable function Ioss (EUDRACT: 2007-000624-40, ClinicalTrials.gov identifier, NCT01137916).

\section{Discusion}

Taking into consideration all these heterogeneous and partly controversial results, variable clinical presentation and behaviour of $\mathrm{AF}$ as well as spontaneous regression and stabilization of disease progression no standard approach for AF can be recommended. AF is a rare and heterogeneous disease that definitely requires individualized treatment to reduce the chance for local tumor control failure. The aims of individualized therapy should include reducing morbidity and function loss and preserving patient quality of life.

Mainstay treatment is surgery particularly for patients with primarily resectable and localized tumors where standard goal is complete resection with negative microscopic margins. Potential mutilation and association with considerable function loss or major morbidity of surgery emphasise that surgical therapy must be performed to what is achievable in terms of margins while preserving functional status for the individual patient.

Attempts to achieve negative margins may result in unnecessary morbidity and may not definitively prevent local recurrence. The consequences of radical excision may be worse than the disease itself.

Radiotherapy is indicated either as adjuvant therapy to surgery with positive margins or as primary therapy for tumors that are recurrent and unresectable or where surgery derived functional/cosmetic loss is unacceptable. Main concerns with radiation therapy are complications-fibrosis as most common and functions impairment. The most serious risk that has to be noted is radiation induced malignancy.

Chemotherapy with cytotoxic agents represents an appropriate choice for patients with unresectable, rapidly and aggressively growing tumors or for patients who are highly symptomatic and/or with life-threatening desmoid tumor. Preferable regime includes pegylated liposomal doxorubicin because of lower toxicity rate. A main concern about chemotherapy is cumulative toxicity partly due to the pharmacological characteristic of the agents and partly due to the prolonged period of application for obtaining treatment response.

Systemic therapy administration, including antihormonal therapy and NSAIDs, for advanced AF disease is justified in cases of recurrent, not life threatening disease in which previous treatments with surgery and radiotherapy did not give response. Although, some studies presented positive results when applying systemic therapy as primary treatment, lack of randomized controlled clinical trials arouses much controversy regarding this issue. Main concerns are duration of treatment to achieving positive response and related to it possible toxicity for certain medication. (In 2004, the U.S. FDA issued a public health advisory recommendation for COX-2 inhibitors (Paper No. T04$61)$. 
Table 1. Imatinib treatment in patients with AF.

\begin{tabular}{|c|c|c|c|c|c|c|c|c|}
\hline Authar & $\begin{array}{l}\text { Na of } \\
\text { patients }\end{array}$ & Sex & $\begin{array}{l}\text { Primay } \alpha \\
\text { reaurent }\end{array}$ & Piortredment & Treatrert & Resporse & $\begin{array}{l}\text { Response } \\
\text { duration }\end{array}$ & TIF \\
\hline Meceata 2002[4B] & 2 & M & reaurent & $\begin{array}{c}\text { surgery, radđherapy, } \\
\text { dematherapy, NSAIDs, } \\
\text { artiestrogen }\end{array}$ & Imatinib800mg/day & $2 \mathbb{R}$ & $\mathrm{NR}$ & $\mathrm{NR}$ \\
\hline Heirich đal.2006 [44] & 19 & $8 \mathrm{~F}, 11 \mathrm{M}$ & reaurent & $\begin{array}{l}\text { surgery, raddherapy, } \\
\text { dhemotherapy, NSAIDS, } \\
\text { artiestrogen }\end{array}$ & Inatinib800my/day & $3 \mathrm{R}, 4 \mathrm{SD}$ & $>12$ manths & 325 cays \\
\hline Heirichđal.2008 [45] & 20 & NR & reaurent & $\mathrm{NR}$ & Imatinib800mg/day & $2 \mathrm{R}, 8 \mathrm{SD}$ & $\mathrm{NR}$ & 277 cays \\
\hline Chugh đal. 2010 [46] & 51 & $37 \mathrm{~F}, 14 \mathrm{M}$ & reaurent & surgery, raddherapy & Imtinib $200-600 \mathrm{mg} / \mathrm{cay}$ & 43SD & 3 yeas & $\mathrm{NR}$ \\
\hline Pend at a. 2011 [47] & 40 & $28 \mathrm{~F}, 12 \mathrm{M}$ & reaurrent & $\begin{array}{c}\text { surgery, radotherpy, } \\
\text { dematherapy, NSAIDs, } \\
\text { artiestrogen }\end{array}$ & Imatinib400mg/day & $1 C R, 3 R, 28 \Phi$ & 2 yeas & $\mathrm{NR}$ \\
\hline
\end{tabular}

Abbreviations F-fende, M-Male,NR- Nbtreported CR-Completeresponse, PRPatid response, SD-Steble d sease, TTF-Time to treatmert failure

Imatinib may be considered as an option in the treatment of recurrent AF with different treatments modalities previously applied as supported by studies-results presented in Table 1. Further clinical trials with translational studies are required to clearly identify the target and better characterize the position of this drug in the sequencing of treatment.
Studies hypothesise that PDGFRB may be the target imatinib-responsive kinase in this disease, but additional studies will be required to confirm this hypothesis and to identify biomarkers predictive of imatinib response/nonresponse in AF tumors and possible genomic mechanisms of activating imatinib sensitive tyrosine kinases. Immunohistochemistry/ immunoblotting evaluations are presented in Table 2.

Table 2: Immunohistochemistrylimmunoblotting evaluations.

\begin{tabular}{|c|c|c|c|c|c|c|c|c|}
\hline Author & $\begin{array}{l}\text { No. of } \\
\text { patients }\end{array}$ & $\begin{array}{l}\text { Primary or } \\
\text { recurrent }\end{array}$ & c-Kit & PDGFR-A & PDGFR-B & p c-Kit & p PDGFR-A & p PDGR-B \\
\hline Mace at a 2002 [43] & 9 & recurrent & $\mathrm{v}$ & $\mathrm{v}$ & $\mathrm{v}$ & NA & NA & NA \\
\hline Heinrich at al.2006 [44] & 19 & recurrent & $x$ & $x$ & v & $x$ & $x$ & $x$ \\
\hline Heinrich at al.2008 [45] & 20 & recurrent & $\mathrm{x}$ & $x$ & v & $\mathrm{x}$ & $x$ & $x$ \\
\hline Chugh at al. 2010 [46] & 51 & recurrent & $\mathrm{v}$ & $\mathrm{v}$ & $\mathrm{v}$ & $\mathrm{x}$ & $\mathrm{x}$ & $x$ \\
\hline
\end{tabular}

Abbreviations: PDGFR: Platelet-derived growth factor receptor; PDGF: Platelet-derived growth factor, p- phosphorilated, v : Positive; X: Negative; NA: not available. 
Although the response rate of AF to imatinib is low, the toxicity profile is favorable and some patients had prolonged PFS. Given the variable behavior of AF and choice of PFS as an endpoint in clinical trials, it is difficult to determine the true benefit of imatinib in patients with AF. The sequencing of imatinib relative to other systemic treatments for AF, which are more toxic, but with higher response rate, is also question of debate. For patients who fail or refuse hormonal or low-dose chemotherapy treatment for recurent AF, it may be reasonable to consider imatinib therapy. For patients in need of rapid shrinkage of their tumor due to disabling symptoms or impending loss of limb or organ damage, aggressive chemotherapy may be preferable. Nevertheless, imatinib is another therapy that could be considered as new option for use in advanced, recurrent AF.

Finally, considering the natural history and heterogeneous biological behaviour of AF, which is often characterized by prolonged periods of stability or even regression, a period of watchful waiting may be the most appropriate management in asymptomatic patients.

Even initial observation may be an adequate action to avoid over treatment.

Conclusion: Given the rarity of this disease, prospective trials and correlative laboratory investigations will require multicenter cooperation. Referral of AF patients to expert teams is the only way to gain sufficiently large experience in a prospective way. Such efforts will be able to identify clinical or molecular criteria by which patients can be selected for each single therapy, longterm observation, or intense multimodality approaches with the vision of keeping patients with this disease alive and preserving their quality of life.

\section{Acknowledgements}

Publication fee for this review article was covered by Ss Cyril and Methodius University Skopje PhD School.

\section{References}

1. Chaudhuri B, Das Gupta TK. Pathology of soft tissue sarcomas. In Das Gupta TK, Chaudhuri PK (eds): Tumors of the Soft Tissues. Stamford, CT: Appleton and Lange, 1998: 63-200.

2. Biermann JS. Desmoid tumors. Curr Treat Options Oncol.
2000; 1:262-266.

3. Clark SK, Phillips RK. Desmoids in familial adenomatous polyposis. Br J Surg. 1996; 83:1494-1504.

4. Giarola M, Wells D, Mondini $P$, et al. Mutations of adenomatous polyposis coli (APC) gene are uncommon in sporadic desmoid tumors. Br J Cancer. 1998;78:582-7.

5. Tejpar S, Nollet F, Li C, et al. Predominance of beta-catenin mutations and beta-catenin dysregulation in sporadic aggressive fibromatosis (desmoid tumor). Oncogene. 1999; 18:6615-20.

6. Mankin HJ, Hornicek FJ, Springfield DS. Extra-abdominal desmoid tumors:a report of 234 cases. J Surg Oncol. 2010; 102: 380-384.

7. Melis M, Zage JS, Sondak VK. Multimodality management of desmoid tumors: How important is a negative surgical margin? J Surg Oncol. 2008; 98: 594-602.

8. Nakayama T, Tsuboyama T, Toguchida J et al. Natural course of desmoidtype fibromatosis. J Orthop Sci. 2008;13:51-55.

9. Nuyttens JJ, Rust PF, Thomas CR, Turrisi AT III. Surgery versus radiation therapy for patients with aggressive fibromatosis or desmoid tumors. A comparative review of 22 articles. Cancer. 2000;88:1517-1523.

10. Ballo MT, Zagars GK, Pollack A, Pisters PW, Pollack RA. Desmoid tumor: Prognostic factors and outcome after surgery, radiation therapy, or combined surgery and radiation therapy. J Clin Oncol. 1999;17:158-167.

11. Spear MA, Jennings LC, Mankin HJ, Spiro IJ, Springfield DS, Gebhardt MC, Rosenberg AE, Efird JT, Suit HD. Individualizing management of aggressive fibromatoses. Int J Radiat Oncol Biol Phys. 1998;40(3):637-45.

12. Stoeckle E, Coindre JM, Longy M, Binh MB, Kantor G, Kind M, de Lara CT, Avril A, Bonichon F, Bui BN. A critical analysis of treatment strategies in desmoid tumours: a review of a series of 106 cases. Eur J Surg Oncol. 2009;35(2):12934.

13. Gronchi A, Casali PG, Mariani L, Lo Vullo S, Colecchia M, Lozza L, Bertulli R, Fiore M, Olmi P, Santinami M, Rosai J. Quality of surgery and outcome in extra-abdominal aggressive fibromatosis: a series of patients surgically treated at a single institution. J Clin Oncol. 2003;21(7):1390-7.

14. Lev D, Kotilingam D, Wei C, Ballo MT, Zagars GK, Pisters PW, Lazar AA, Patel SR, Benjamin RS, Pollock RE. Optimizing treatment of desmoid tumors. J Clin Oncol. 2007;25(13):178591.

15. Lewis JJ, Boland PJ, Leung DH, Woodruff JM, Brennan MF. The enigma of desmoid tumors. Ann Surg. 1999;229(6):866-72; discussion 872-3.

16. Merchant NB, Lewis JJ, Woodruff JM, Leung DH, Brennan 
MF. Extremity and trunk desmoid tumors: a multifactorial analysis of outcome. Cancer. 1999;86(10):2045-52.

17. Shido $Y$, Nishida $Y$, Nakashima $H$, Katagiri $H$, Sugiura $H$, Yamada $Y$, Ishiguro N. Surgical treatment for local control of extremity and trunk desmoid tumors. Arch Orthop Trauma Surg. 2009;129(7):929-33.

18. Bonvalot S, Eldweny $\mathrm{H}, \mathrm{Haddad} \mathrm{V}$ et al. Extra-abdominal primary fibromatosis: Aggressive management could be avoided in a subgroup of patients. Eur J Surg Oncol. 2008; 34: $462-468$.

19. Janinis J, Patriki M, Vini L et al. The pharmacological treatment of aggressive fibromatosis: A systematic review. Ann Oncol. 2003; 14:181-190.

20. Kasper B, Ströbel P, Hohenberger P. Desmoid tumors: clinical features and treatment options for advanced disease. Oncologist. 2011;16(5):682-93.

21. Nuyttens JJ, Rust PF, Thomas CR Jr et al. Surgery versus radiation therapy for patients with aggressive fibromatosis or desmoid tumors: A comparative review of 22 articles. Cancer. 2000; 88:1517-1523.

22. Baumert BG, Spahr MO, von Hochstetter A et al. The impact of radiotherapy in the treatment of desmoid tumours. An international survey of 110 patients. A study of the Rare Cancer Network. Radiat Oncol. 2007; 2:1-12.

23. Gluck I, Griffith KA, Biermann S et al. Role of radiotherapy in the management of desmoid tumors. Int J Radiat Oncol Biol Phys. 2011; 80: 787-792.

24. Waddell WR, Gerner RE. Indomethacin and ascorbate inhibit desmoid tumours. J Surg Oncol. 1980; 15: 85-90.

25. Hansmann A, Adolph C, Vogel T et al. High-dose tamoxifen and sulindac as firstline treatment for desmoid tumors. Cancer. 2004;100:612-620.

26. Tsukada K, Church JM, Jagelman DJ et al. Noncytotoxic therapy for intra-abdominal desmoid tumor in patients with familial adenomatous polyposis. Dis Colon Rectum. 1992; 35: 29-33.

27. Poon R, Smits R, Li C, Jagmohan-Changur S, Kong M, Cheon S, Yu C, Fodde R, Alman BA. Cyclooxygenase-two (COX-2) modulates proliferation in aggressive fibromatosis (desmoid tumor). Oncogene. 2001;20(4):451-60.

28. Signoroni S, Frattini M, Negri T, Pastore E, Tamborini E, Casieri P, Orsenigo M, Da Riva L, Radice P, Sala P, Gronchi A, Bertario L, Pierotti MA, Pilotti S. Cyclooxygenase-2 and platelet-derived growth factor receptors as potential targets in treating aggressive fibromatosis. Clin Cancer Res. 2007;13(17):5034-40. Erratum in: Clin Cancer Res. 2008;14(13):4354.

29. Francis WP, Zippel D, Mack LA, DiFrancesco LM, Kurien E, Schachar NS, Temple WJ. Desmoids: a revelation in biology and treatment. Ann Surg Oncol. 2009;16(6):1650-4.

30. Eisenhauer EA, Therasse P, Bogaerts J, Schwartz LH, Sargent D, Ford R, Dancey J, Arbuck S, Gwyther S, Mooney M, Rubinstein L, Shankar L, Dodd L, Kaplan R, Lacombe D, Verweij J. New response evaluation criteria in solid tumours: revised RECIST guideline (version 1.1). Eur J Cancer. 2009;45(2):228-47.

31. Nishida Y, Tsukushi S, Shido Y, et al. Successful treatment with meloxicam, a cyclooxygenase-2 inhibitor, of patients with extra-abdominal desmoid tumors: a pilot study. J Clin Oncol. 2010; 28: e107-e109.

32. Hansmann A, Adolph C, Vogel T, et al. High-dose tamoxifen and sulindac as first-line treatment for desmoid tumors. Cancer. 2004; 100:612-620.

33. Brooks MD, Ebbs SR, Colletta AA et al. Desmoid tumors treated with triphenylethylenes. Eur J Cancer. 1992;28A: 10141018.

34. Benson JR, Mokbel K, Baum M. Management of desmoid tumors including a case report of toremifene. Ann Oncol. 1994; 5:173-177.

35. Lanari A. Effect of progesterone on desmoid tumors (aggressive fibromatosis). N Engl J Med. 1983; 309: 1523.

36. Waddell WR, Kirsch WM. Testolactone, sulindac, warfarin, and vitamin K1 for unresectable desmoid tumors. Am J Surg. 1991;161:416- 421.

37. Patel SR, Benjamin RS. Desmoid tumors respond to chemotherapy: defying the dogma in oncology. J Clin Oncol. 2006; 24: 11-12.

38. Constantinidou A, Jones RL, Scurr M, et al. Pegylated liposomal doxorubicin, an effective, well-tolerated treatment for refractory aggressive fibromatosis. Eur J Cancer. 2009;45:2930-2934.

39. De Camargo VP, Keohan ML, D'Adamo DR et al. Clinical outcomes of systemic therapy for patients with deep fibromatosis (desmoid tumor). Cancer. 2010;116:2258-2265.

40. Garbay D, Le Cesne A, Penel N et al. Chemotherapy in patients with desmoid tumors: a study from the French Sarcoma Group (FSG). Ann Oncol. 2012; 23:182-186.

41. Skapek SX, Ferguson WS, Granowetter Let al. Vinblastine and methotrexate for desmoid fibromatosis in children: Results of a Pediatric Oncology Group phase II trial. J Clin Oncol. 2007; 25:501-506.

42. Azzarelli A, Casali P, Fissi S et al. Effective control of advanced aggressive fibromatosis with chemotherapy. Eight years experience with methotrexate and vinblastine in 27 patients. Proceedings Fourth Annual Meeting Connective Tissue Oncology Society, Milan, 1998.

43. Mace J, Sybil Biermann J, Sondak V, et al. Response of 
extraabdominal desmoid tumors to therapy with imatinib mesylate. Cancer. 2002; 95:2373-9.

44. Heinrich MC, McArthur GA, Demetri GD et al. Clinical and molecular studies of the effect of imatinib on advanced aggressive fibromatosis (desmoid tumour). J Clin Oncol. 2006; 24:1195-1203.

45. Heinrich MC, Joensuu H, Demetri GD et al. Phase II, openlabel study evaluating the activity of imatinib in treating lifethreatening malignancies known to be associated with imatinibsensitive tyrosine kinases. Clin Cancer Res. 2008;14:27172725.

46. Chugh R, Wathen JK, Patel SR et al. Efficacy of imatinib in aggressive fibromatosis: results of a phase II multicenter Sarcoma Alliance for Research through Collaboration (SARC) trial. Clin Cancer Res. 2010;16:4884-4891.
47. Penel N, Le Cesne A, Bui BN et al. Imatinib for progressive and recurrent aggressive fibromatosis (desmoid tumors): an FNCLCC/French Sarcoma Group phase II trial with a longterm follow-up. Ann Oncol. 2011; 22: 452-457.

48. Kurtz JE, Asmane I, Voegeli AC et al. A V530I mutation in C-KIT exon 10 is associated to imatinib response in extraabdominal aggressive fibromatosis. Sarcoma. 2010; 2010: 458156.

49. Leithner A, Gapp M, Radl R et al. Immunohistochemical analysis of desmoid tumours. J Clin Pathol. 2005; 58:11521156.

50. Dufresne A, Bertucci F, Penel $N$ et al. Identification of biological factors predictive of response to imatinib mesylate in aggressive fibromatosis. Br J Cancer. 2010; 103: 482-485. 